\title{
A New Method for Optimal Placement of TCSC Based on Sensitivity Analysis for Congestion Management
}

\author{
Abouzar Samimi ${ }^{1}$, Peyman Naderi ${ }^{2}$ \\ ${ }^{1}$ Department of Electrical Engineering, Islamic Azad University Boroujerd Branch, Boroujerd, Iran; ${ }^{2}$ Department of Electrical Engi- \\ neering, Shahid Rajaee University, Tehran, Iran. \\ Email: abouzarsamimi@iust.ac.ir,naderi@ee.kntu.ac.ir
}

Received June $5^{\text {th }}, 2011$; revised December $7^{\text {th }}, 2011$; accepted December $14^{\text {th }}, 2011$

\begin{abstract}
In this paper a new method has been proposed to determine optimal location and best setting of Thyristor Controlled Series Compensator (TCSC). Seeking the best place is performed using the sensitivity analysis and optimum setting of TCSC is managed using the genetic algorithm. The configuration of a typical TCSC from a steady-state perspective is the fixed capacitor with a thyristor controlled reactor (TCR). The effect of TCSC on the network can be modeled as a controllable reactance inserted in the related transmission line. This paper employs the DIgSILENT simulator and the DPL as a programming tool of the DIgSILENT to show the validity of the proposed method. The effectiveness of suggested approach has been tested on IEEE 14-bus system.
\end{abstract}

Keywords: TCSC; Optimal Placement; Sensitivity Analysis; Genetic Algorithm

\section{Introduction}

In recent years, with increasing in development of power networks, the economical operation of power system is more considered. Because of deregulation and restructuring of the electricity markets use of Flexible AC Transmission Systems (FACTS) devices is inevitable. The maximum capability of power systems can be exploited by means of FACTS devices. Nowadays, development of power electronics switches causes reduction in the cost of FACTS and therefore application of FACTS devices especially in distribution networks is more economical.

Because of the economical considerations, installation of FACTS controller in all of the buses or the lines is impossible and unnecessary. There are several methods for finding optimal locations of FACTS devices in power systems [1-7].

In [1], a sensitivity based method has been suggested to optimally locate the Thyristor Controlled Series Compensator (TCSC) and Unified Power Flow Controller (UPFC) for enhancing the system security under different operating conditions and at optimal settings of FACTS parameters. The DC power flow equations have been employed for calculating the sensitivity indices. In [2], a genetic algorithm (GA) based method is used to determine the optimal sitting of FACTS controller in power system. The fitness function is to minimize the generation cost. In [3], the genetic algorithm is used to seek the optimal location of multi-type FACTS devices in a power system. The optimizations are performed on three parameters: the location of the devices, their types, and their values. In [4], the Tabu Search (TS) method is used to solve the combinatorial (i.e. to determine number and location) problem of FACTS device allocation. Reference [5] compares three heuristic methods, simulated annealing (SA), TS and GA, applied to the optimal location of FACTS devices in order to enhance the system security. The objective function is based on indices quantifying the severity of the contingencies in terms of branch loading and voltage levels. The three methods lead to similar results, but generally TS and GA converge faster than SA to an optimal solution. In [6], a real power flow performance sensitivity index has been proposed to decide optimal location of FACTS controllers. In [7], extended voltage phasors approach (EVPA) is proposed for placement of FACTS controllers in power systems within the voltage stability viewpoint.

In this paper a new method has been proposed to optimally locate TCSC in power systems. The suggested approach is composed of sensitivity analysis and the genetic algorithm. Finding the best place for TCSC is performed using the sensitivity analysis and sizing of TCSC is managed using the genetic algorithm. The IEEE 14bus system has been applied to test the suggested algorithm. The rest of the paper is organized as follows. Section 2 presents the modeling of the TCSC adapted for this study. The search space in optimal placement of series 
capacitive compensators in real power systems is usually sizable. Use of the approaches like sensitivity analysis can reduce the search space. In Section 3 some sensitivity indices have been presented. The best setting of TCSC is performed by genetic algorithm in Section 4. Finally numerical results along with some observations and discussions are presented in Section 4. DIgSILENT software which contains a powerful programming language called DPL ${ }^{1}$ has been prepared required facilities to execute the proposed algorithms and corresponding simulations.

\section{TCSC Modeling}

The IEEE defines the TCSC as a capacitive reactance compensator which consists of three main components: capacitor bank $C$, bypass inductor $L$ and bidirectional thyristors SCR1 and SCR2. Series capacitive compensation has been used to increase line power transfer as well as to enhance system stability. Figure 1 shows the main circuit of a TCSC.

The firing angles of the thyristors are controlled to adjust the TCSC reactance according to the system control algorithm, normally in response to some system parameter variations. According to the variation of the thyristor firing angle or conduction angle, this process can be modeled as a fast switch between corresponding reactance offered to the power system. Assuming that the total current passing through the TCSC is sinusoidal, the equivalent reactance at the fundamental frequency can be represented as a variable reactance $X_{T C S C}$. The TCSC can be controlled to work either in the capacitive or the inductive zones avoiding steady state resonance. There exists a steady-state relationship between the firing angle and the reactance $X_{T C S C}$. This relationship can be described by the following equation [8]:

$$
X_{T C S C}(\alpha)=\frac{X_{C} X_{l}(\alpha)}{X_{l}(\alpha)-X_{C}}
$$

where,

$$
X_{l}(\alpha)=X_{L} \frac{\pi}{\pi-2 \alpha-\sin \alpha}
$$

$\alpha$ is the firing angle, $X_{L}$ is the reactance of the inductor and $X_{l}$ is the effective reactance of the inductor at firing

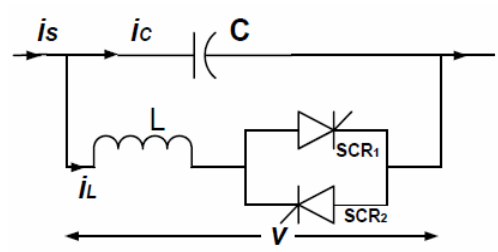

Figure 1. Configuration of a TCSC.

${ }^{1}$ DIgSILENT Programming Language. angle. In this paper the TCSC is taken as continuous varying capacitor. The effective series transmission impedance is given by:

$$
X_{\text {eff }}=(1-k) \cdot X
$$

where $k$ is the degree of series compensation

$$
k=\frac{X_{T C S C}}{X} \quad 0 \leq k \leq 10
$$

In the simulations of this paper, only the capacitive region has been used. Hence the compensation level varies from zero to the maximum level of 0.7 . Figure 2 shows a transmission line with a TCSC.

In some references a static Power Injection Model (PIM) of the TCSC has been presented. The injection model represents the TCSC as a device that injects certain amount of active and reactive power in a node [1].

$$
\begin{gathered}
P_{i c}=V_{i}^{2} G_{i j}^{\prime}-V_{i} V_{j}\left[G_{i j}^{\prime} \cos \left(\delta_{i j}\right)+B_{i j}^{\prime} \sin \left(\delta_{i j}\right)\right] \\
Q_{i c}=-V_{i}^{2}\left(B_{i j}^{\prime}+B_{s h}\right)-V_{i} V_{j}\left[G_{i j}^{\prime} \sin \left(\delta_{i j}\right)-B_{i j}^{\prime} \cos \left(\delta_{i j}\right)\right] \\
P_{j c}=V_{j}^{2} G_{i j}^{\prime}-V_{i} V_{j}\left[G_{i j}^{\prime} \cos \left(\delta_{i j}\right)-B_{i j}^{\prime} \sin \left(\delta_{i j}\right)\right] \\
Q_{j c}=-V_{j}^{2}\left(B_{i j}^{\prime}+B_{s h}\right)+V_{i} V_{j}\left[G_{i j}^{\prime} \sin \left(\delta_{i j}\right)+B_{i j}^{\prime} \cos \left(\delta_{i j}\right)\right]
\end{gathered}
$$

where,

$$
\begin{gathered}
G_{i j}^{\prime}=\frac{r_{i j}}{r_{i j}^{2}+\left(x_{i j}-x_{c}\right)^{2}} \\
B_{i j}^{\prime}=\frac{-\left(x_{i j}-x_{c}\right)}{r_{i j}^{2}+\left(x_{i j}-x_{c}\right)^{2}}
\end{gathered}
$$

\section{Sensitivity Analysis for Optimal Placement of TCSC}

Many sensitivity performance indices have been proposed for the analysis of power systems. There are some sensitivity indices which have the most attraction for optimal placement of series compensators.

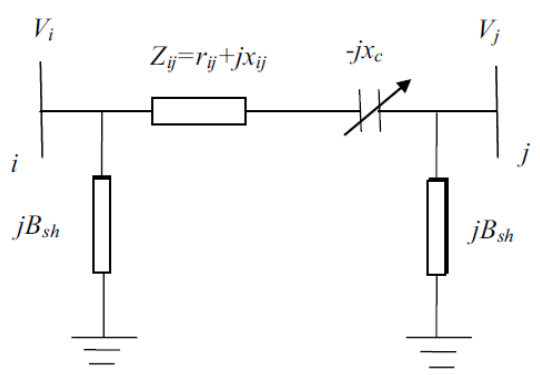

Figure 2. Static model of line with TCSC. 


\subsection{Sensitivity Analysis for Optimal Placement of TCSC}

Here we look at a method based on the sensitivity of the total system reactive power loss with respect to the control variable of the TCSC. For TCSC placed between buses $i$ and $j$, we consider net line series reactance as a control parameter. Loss sensitivity with respect to control parameter of TCSC placed between buses $i$ and $j$ can be written as [9]:

$$
a_{i j}=\frac{\partial Q_{L}}{\partial x_{i j}}=\left[V_{i}^{2}+V_{j}^{2}-2 V_{i} V_{j} \cos \delta_{i j}\right] \cdot \frac{r_{i j}^{2}-x_{i j}^{2}}{\left(r_{i j}^{2}+x_{i j}^{2}\right)^{2}}
$$

\subsection{Sensitivity Analysis for Optimal Placement of TCSC}

The sensitivity $a_{c}^{j}$ of transmission loss $\left(P_{L j}\right)$ on a series compensated line- $j$ with respect to series capacitive reactance $\left(X_{c j}\right)$ is defined as follows [10]:

$$
a_{c}^{j}=\left.\frac{\partial P_{L k}}{\partial X_{c j}}\right|_{X_{c j}=0}=-2\left[V_{i}^{2}+V_{j}^{2}-2 V_{i} V_{j} \cos \delta_{i j}\right] G_{i j} B_{i j}
$$

\subsection{Total System Loss Sensitivity Index}

The real power loss of a system having $N$ bus is:

$$
P_{L T}^{\prime}=\sum_{j=1}^{N} \sum_{k=1}^{N}\left[\alpha_{j k}\left(P_{j} P_{k}+Q_{j} Q_{k}\right)+\beta_{j k}\left(Q_{j} P_{k}-P_{j} Q_{k}\right)\right]
$$

where $P_{j}$ and $Q_{j}$ respectively, are the real and reactive power injected at bus- $j$ and $\alpha, \beta$ are the loss coefficients defined by:

$$
\begin{gathered}
\alpha_{j k}=\frac{r_{j k}}{V_{j} V_{k}} \cos \left(\delta_{j}-\delta_{k}\right) \\
\beta_{j k}=\frac{r_{j k}}{V_{j} V_{k}} \sin \left(\delta_{j}-\delta_{k}\right)
\end{gathered}
$$

where $r_{j k}$ is the real part of the $j-k^{\text {th }}$ element of $Z_{b u s}$ matrix. Using power injection model of FACTS this total loss if FACTS device, one at a time is used, can be written as [10]:

$$
P_{L T}=P_{L T}^{\prime}-\left(P_{i c}+P_{j c}\right)
$$

The total system real power loss sensitivity factors with respect to the parameters of TCSC placed at line- $k$ can be defined as [10]:

$$
b_{C}^{k}=\left.\frac{\partial P_{L T}}{\partial X_{c k}}\right|_{X_{c k}=0}
$$

Consider a line- $k$ connected between bus- $i$ and bus- $j$.
The total system loss sensitivity with respect to TCSC can be derived as given below [10]:

$$
\begin{aligned}
b_{c}^{k}= & \left.\frac{\partial P_{L T}}{\partial P_{i}} \frac{\partial P_{i}}{\partial X_{c k}}\right|_{X_{c k}=0}+\left.\frac{\partial P_{L T}}{\partial P_{j}} \frac{\partial P_{j}}{\partial X_{c k}}\right|_{X_{c k}=0} \\
& +\left.\frac{\partial P_{L T}}{\partial Q_{i}} \frac{\partial Q_{i}}{\partial X_{c k}}\right|_{X_{c k}=0}+\left.\frac{\partial P_{L T}}{\partial Q_{j}} \frac{\partial Q_{j}}{\partial X_{c k}}\right|_{X_{c k}=0} \\
& -\left.\left(\frac{\partial P_{i c}}{\partial X_{c k}}+\frac{\partial P_{j c}}{\partial X_{c k}}\right)\right|_{X_{c k}=0}
\end{aligned}
$$

where,

$$
\begin{gathered}
\frac{\partial P_{L T}}{\partial P_{i}}=2 \sum_{m=1}^{N}\left[\alpha_{i m} P_{m}-\beta_{i m} Q_{m}\right] \\
\frac{\partial P_{L T}}{\partial Q_{i}}=2 \sum_{m=1}^{N}\left[\alpha_{i m} Q_{m}+\beta_{i m} P_{m}\right] \\
\left.\frac{\partial P_{i}}{\partial X_{c k}}\right|_{X_{c k}=0}=\left.\frac{\partial P_{i c}}{\partial X_{c k}}\right|_{X_{c k}=0}=-2\left[V_{i}^{2}-V_{i} V_{j} \cos \delta_{i j}\right] \\
\times \frac{r_{i j} x_{i j}}{\left(r_{i j}^{2}+x_{i j}^{2}\right)^{2}}-V_{i} V_{j} \sin \delta_{i j} \frac{r_{i j}^{2}-x_{i j}^{2}}{\left(r_{i j}^{2}+x_{i j}^{2}\right)^{2}} \\
=\left.\frac{\partial P_{i c}}{\partial X_{c k}}\right|_{X_{c k}=0}=-2\left[V_{i}^{2}-V_{i} V_{j} \cos \delta_{i j}\right] \\
\times \frac{r_{i j} x_{i j}}{\left(r_{i j}^{2}+x_{i j}^{2}\right)^{2}}-V_{i} V_{j} \sin \delta_{i j} \frac{r_{i j}^{2}-x_{i j}^{2}}{\left(r_{i j}^{2}+x_{i j}^{2}\right)^{2}}
\end{gathered}
$$

\subsection{Real Power Flow Sensitivity Index}

In this section, a new real power flow sensitivity index with respect to the parameter of TCSC placed in line $j$ is introduced as:

$$
S I_{j}=\sum_{m=1}^{N_{l}} \beta_{m} \frac{\partial P_{m}}{\partial X_{j}}
$$

In this index, TCSC has been modeled as a variable series capacitive reactance $X_{T C S C}$. Therefore, the total line reactance decreases. This index demonstrates the sum of variation of real power flow in all lines with respect to the change of reactance of line $j . \quad \beta_{m}$ is a weighted factor which can be selected higher for congested lines. In this study $\beta_{m}$ is selected five for congested lines.

Calculating of the $S I_{j}$ index can be performed using DC power flow equations [11]. But for accurate calculation, this index is computed using $\mathrm{AC}$ power flow. For this purpose, the line reactance would be very little changed around the operating point subject to the other conditions are fixed. Hence $S I_{j}$ can be rewritten as: 


$$
S I_{j}=\left.\sum_{m=1}^{N_{l}} \beta_{m} \frac{\Delta P_{m}}{\Delta X_{j}}\right|_{\Delta X_{j} \rightarrow 0}
$$

This index is calculated for all the lines. After that $S I-$ min and $S I_{\max }$ are specified by sorting the $S I$ values, normalized real power flow index is defined as:

$$
S I n_{j}=\frac{S I_{j}-S I_{\min }}{S I_{\max }-S I_{\min }}
$$

TCSC must be placed in a line having the most positive sensitivity index.

\section{Optimal Setting of TCSC Using the Genetic Algorithm}

The genetic algorithm has been used to find the optimum sizing of TCSCs. Genetic algorithms are based on the mechanisms of natural selection. The principles and details of the genetic algorithm have been presented in many references.

\subsection{Objective Function}

The objective function has been made of the severity of the system loading by the following relationship:

Minimize:

$$
F=\sum_{m=1}^{N_{l}}\left(\frac{S_{L m}}{S_{L m}^{\max }}\right)^{2 n}
$$

where,

$P_{L m}:$ Apparent power flow in line $m$

$P_{L m}^{\max }:$ The rated capacity of line- $m$

$N_{l}$ : The number of power system lines

The objective function $F$ will be small when all the lines are within their limits and reach a high value when there are overloads. Thus, it provides a good measure of severity of the line overloads for given state of the power system. Most of the works on contingency selection algorithms utilize the second order performance indices which, in general, suffer from masking effects. The lack of discrimination, in which the performance index for a case with many small violations may be comparable in value to the index for a case with one huge violation, is known as masking effect. By most of the operational standards, the system with one huge violation is much more severe than that with many small violations. Masking effect to some extent can be avoided using higher order performance indices. However, in this study, the value of exponent has been taken as 2 .

\subsection{Initial Population}

Some responses as chromosomes of initial population must be created for starting algorithm. The length of each chromosome (the number of genes formed a chromosome) is the number of decimal places. In fact every gene is a number between $0-9$ and each chromosome shows exact the degree of series compensation $(k)$ for TCSC.

\subsection{Selection Operator}

The best solutions in the current population are selected by roulette wheel technique.

\subsection{Crossover Operator}

Two random chromosomes in the middle generation are selected. Then a random number $(n)$ between 1 to the length of chromosome are selected and pairs of selected chromosomes from $n$-th gene to later are swapped to each other to produce new chromosomes.

\subsection{Mutation Operator}

To test each element for fitness and to avoid algorithm stopping at a local optimum some solutions are also randomly modified. Therefore a chromosome is selected randomly, then some of it genes are replaced with another random numbers.

\section{Numerical Results}

The case study for examination of the proposed algorithm is the IEEE 14-bus system.

All the loads of IEEE 14-bus system have been modeled by the following polynomial equations:

$$
\begin{aligned}
& P=P_{0}\left(\frac{V}{V_{0}}\right)^{\alpha} \\
& Q=Q_{0}\left(\frac{V}{V_{0}}\right)^{\beta}
\end{aligned}
$$

where $P_{0}$ and $Q_{0}$ stand for the real and reactive powers consumed at a reference voltage $V_{0}$. In this study the value of exponents have been taken as $\alpha=1.6$ and $\beta=1.8$. In addition a $30 \%$ increasing coefficient for active and reactive power loads rather than the base values is considered.

Having been calculated the real power flow sensitivity indices, the results are shown in Table 1 Regarding to the results shown in Table 1, line $1-5$ has the most positive sensitivity index. Therefore this line is selected for installing of TCSC. Analyzing the results of loading, it was clear this place is close to the most congested line 1 2. Figure 3 shows situation of this place on the network. The degree of compensation $(k)$ is calculated as 0.59 by implementing the genetic algorithm.

Table 2 shows the loading of lines in the base state and after placing TCSC in line $1-5$. The most congested 
Table 1. The real power flow sensitivity indices.

\begin{tabular}{ccc}
\hline Line & $S I(j)$ & $\operatorname{SIn}(j)$ \\
\hline $1-2$ & -24.2878 & 0 \\
$1-5$ & 12.4146 & 1 \\
$10-11$ & -0.0126 & 0.6614 \\
$12-13$ & -0.0218 & 0.6612 \\
$12-6$ & -0.1296 & 0.6582 \\
$14-13$ & -0.0110 & 0.6614 \\
$2-5$ & -5.0649 & 0.5238 \\
$3-2$ & 1.3038 & 0.6973 \\
$3-4$ & -0.3280 & 0.6528 \\
$4-2$ & -2.6979 & 0.5882 \\
$4-5$ & 2.3310 & 0.7253 \\
$6-11$ & 0.0074 & 0.6620 \\
$6-13$ & 0.3161 & 0.6704 \\
$7-8$ & 0.0132 & 0.6621 \\
$9-10$ & -0.0588 & 0.6601 \\
$9-14$ & 0.0117 & 0.6621 \\
$9-7$ & -0.3549 & 0.6521 \\
\hline
\end{tabular}

Table 2. Loading of lines before and after placing TCSC.

\begin{tabular}{ccccc}
\hline Line & $\begin{array}{c}\text { Rated Voltage } \\
(\mathrm{kV})\end{array}$ & $\begin{array}{c}\text { Rating } \\
(\text { MVA) }\end{array}$ & $\begin{array}{c}\text { \% Loading } \\
\text { without TCSC }\end{array}$ & $\begin{array}{c}\text { \% Loading } \\
\text { with TCSC }\end{array}$ \\
\hline $1-2$ & 132 & 150 & 138.5 & 106.2 \\
$1-5$ & 132 & 150 & 65.5 & 97.4 \\
$10-11$ & 400 & 50 & 15.7 & 19.1 \\
$12-13$ & 400 & 50 & 4.0 & 4.4 \\
$12-6$ & 400 & 50 & 22.2 & 22.4 \\
$14-13$ & 400 & 50 & 15.7 & 18.0 \\
$2-5$ & 132 & 150 & 35.3 & 21.8 \\
$3-2$ & 132 & 150 & 63.5 & 58.1 \\
$3-4$ & 132 & 150 & 22.8 & 28.4 \\
$4-2$ & 132 & 150 & 48.2 & 37.7 \\
$4-5$ & 132 & 150 & 56.6 & 70.6 \\
$6-11$ & 400 & 50 & 20.8 & 24.3 \\
$6-13$ & 400 & 50 & 51.1 & 52.3 \\
$7-8$ & 400 & 60 & 40.2 & 40.0 \\
$9-10$ & 400 & 50 & 33.2 & 33.3 \\
$9-14$ & 400 & 50 & 35.8 & 34.8 \\
$9-7$ & 400 & 75 & 17.2 & 17.3 \\
\hline
\end{tabular}

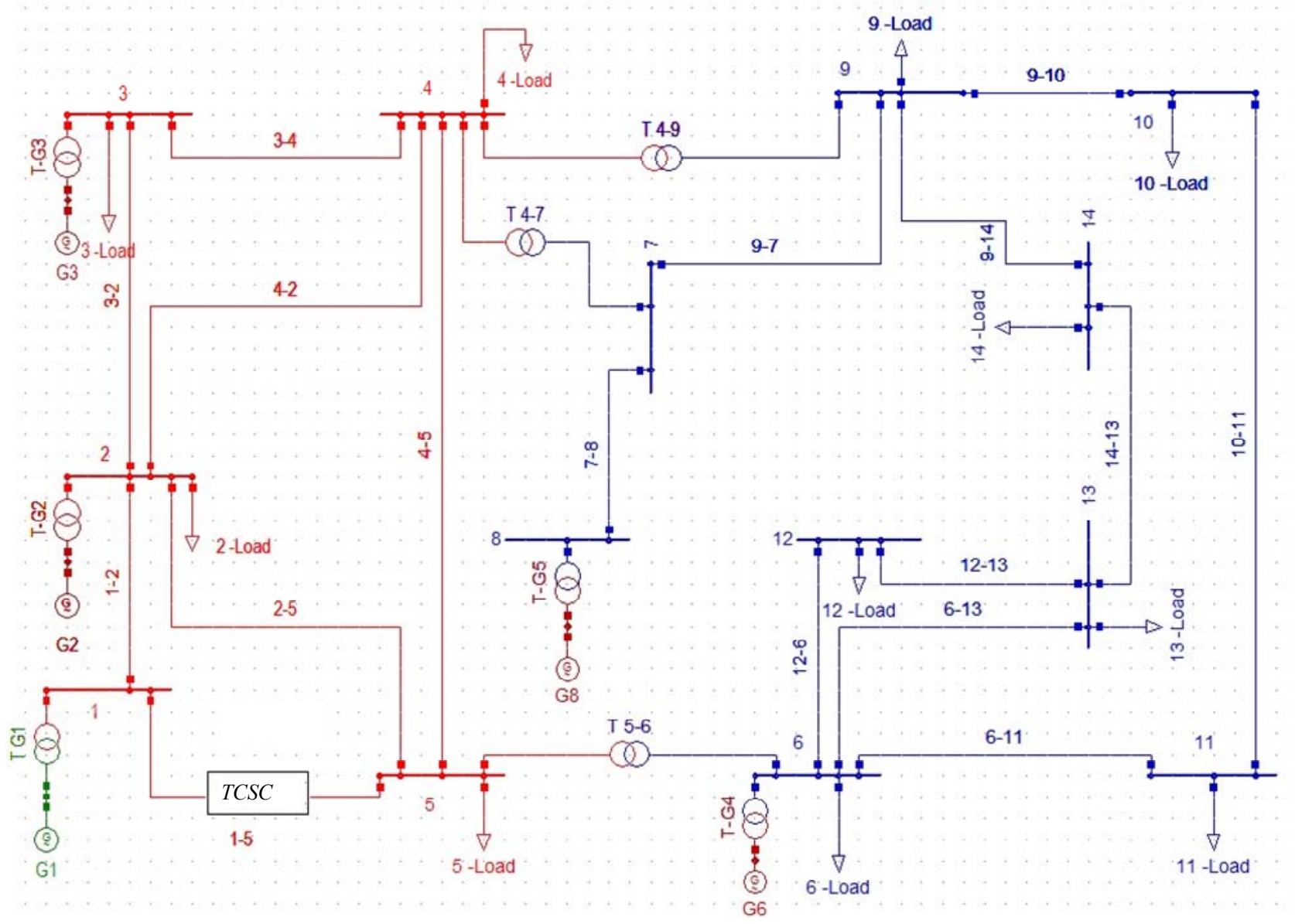

Figure 3. the IEEE 14-bus system in DIgSILENT. 
line has a $138.5 \%$ loading in the base state. After installing TCSC in line 1 - 5 the loading of line 1 - 2 decreases to $106.2 \%$ in exchange for a loading increment of line 1 5 and line $4-5$. As previously mentioned, by most of the operational standards, the system with one huge violation is much more severe than that with many small violations. As it is seen in Table 2, the loading changes of other lines are negligible. The result of optimal placement of $T C S C$ via suggested approach corresponds to the results

Table 3. Voltage magnitude of buses before and after placing TCSC.

\begin{tabular}{ccc}
\hline Bus & Voltage in p.u. without TCSC & Voltage in p.u. with TCSC \\
\hline 1 & 1.060 & 1.060 \\
2 & 1.021 & 1.027 \\
3 & 0.971 & 0.976 \\
4 & 0.971 & 0.976 \\
5 & 0.986 & 0.990 \\
6 & 0.918 & 0.922 \\
7 & 0.952 & 0.957 \\
8 & 0.995 & 0.999 \\
9 & 0.939 & 0.943 \\
10 & 0.924 & 0.928 \\
11 & 0.916 & 0.920 \\
12 & 0.898 & 0.902 \\
13 & 0.894 & 0.898 \\
14 & 0.892 & 0.896 \\
\hline
\end{tabular}

of other approaches in other references [12,13]. Therefore the validity of the proposed method is confirmed.

Bus voltage level before and after compensation process is shown in Table 3. In spite of effective relief of congestion, it is clear the improvement of voltage stability is negligible and there is impermissible voltage drop at some of the buses.

Other results of load flow calculation before and after installing of TCSC are shown in Table 4.

\section{Conclusion}

In this paper a new method has been proposed to optimally locate TCSC in power sytems. The suggested approach is composed of sensitivity analysis and genetic

Table 4. Results of load flow calculation before and after compensation with TCSC.

\begin{tabular}{lcc}
\hline \multicolumn{1}{c}{ Parameter } & Without TCSC & With TCSC \\
\hline Active Power Generation (MW) & 362.25 & 363.36 \\
Reactive Power Generation (MVAr) & 162.77 & 142.82 \\
Active Losses (MW) & 25.55 & 26.66 \\
Reactive Losses (MVAr) & 62.15 & 42.2 \\
$\% \mathrm{P}_{\text {loss }}$ & $\% 7.05$ & $\% 7.34$ \\
$F=\sum_{m=1}^{N_{l}}\left(\frac{S_{L m}}{S_{L m}^{\max }}\right)^{2 n}$ & 4.335 & 2.697 \\
\hline
\end{tabular}

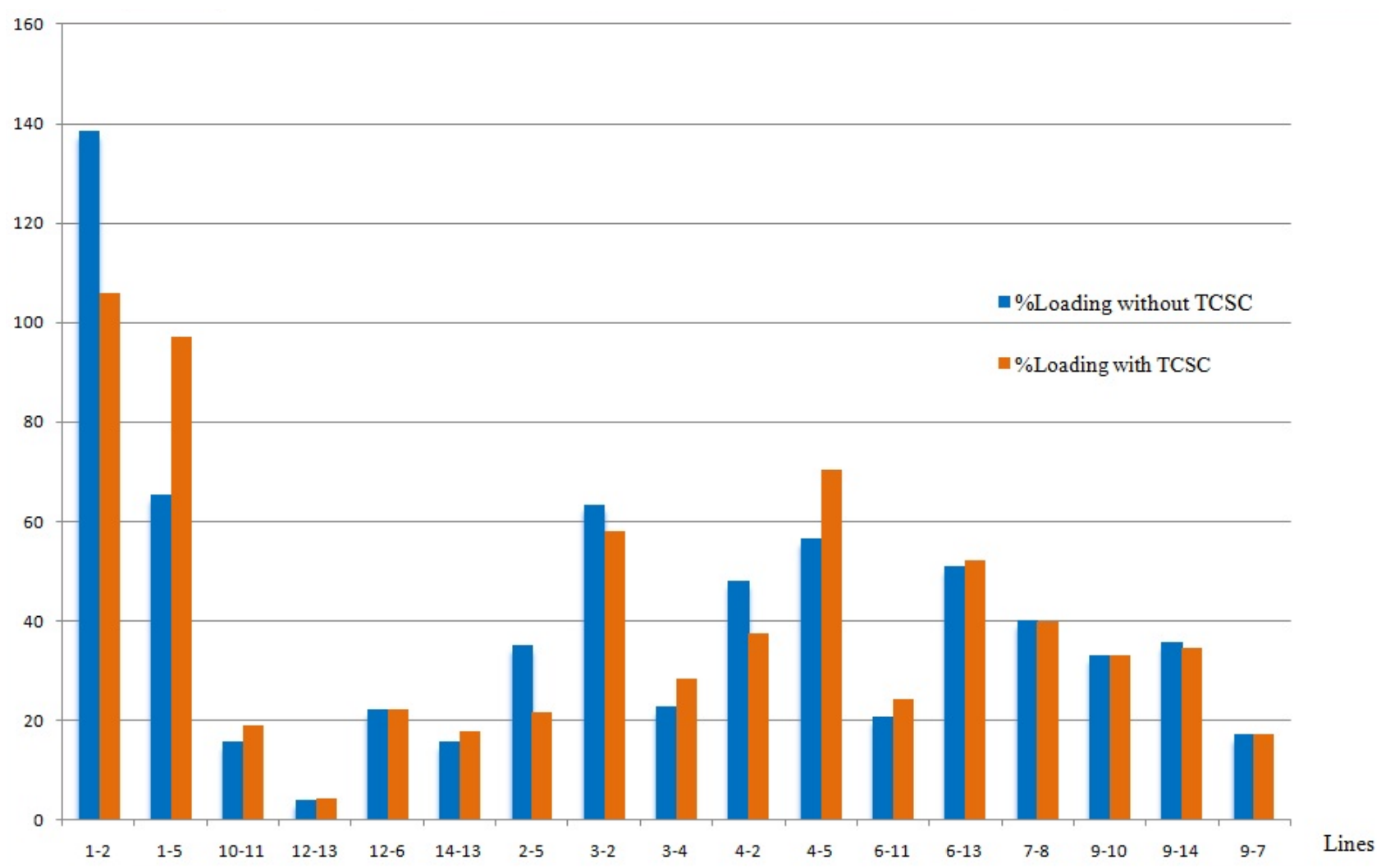

Figure 4. Loading profile before and after compensation. 
algorithm. First, the appropriate modeling of the TCSC has been presented. After introducing some sensitivity indices, sensitivity analysis approach has been utilized to find optimal placement of series compensators. In this process, a real power flow sensitivity index has been presented. Then the setting of TCSC has been defined by GA. The objective function has been made of the severity of the system loading. The result of load flow calculation before and after compensation process shows reduction of loading in congested lines.

\section{REFERENCES}

[1] J. G. Singh, S. N. Singh and S. C. Srivastava, "Enhancement of Power System Security through Optimal Placement of TCSC and UPFC," Power Engineering Society General Meeting, Tampa, 24-28 June 2007, pp. 1-6.

[2] H. C. Leung and T. S. Chung, "Optimal Placement of FACTS Controller in Power System by a Genetic-Based Algorithm," Proceedings of the IEEE International Conference on Power Electronics and Drive Systems, 27-29 July 1999, pp. 833-836.

[3] S. Gerbex, R. Cherkaoui and A. J. Germond, "Optimal Location of Multi-Type FACTS Devices in a Power System by Means of Genetic Algorithms," IEEE Transactions on Power Systems, Vol. 16, No. 3, 2001, pp. 537-544. doi:10.1109/59.932292

[4] H. Mori and Y. Goto, "A Parallel Tabu Search Based Method for Determining Optimal Allocation of FACTS in Power Systems," Proceedings of the International Conference on Power System Technology, Perth, 4-7 December 2000, pp. 1077-1082. doi:10.1109/ICPST.2000.897170

[5] S. Gerbex, R. Cherkaoui and A. J. Germond, "Optimal Location of FACTS Devices to Enhance Power System Security," Proceedings of the Conference on Power Technology, Bologna, 23-26 June 2003, p. 7.

\section{doi:10.1109/PTC.2003.1304363}

[6] J. G. Singh, S. N. Singh and S. C. Srivastava, "Enhancement of Power System Security through Optimal Placement of TCSC and UPFC," Power Engineering Society General Meeting, Tampa, 24-28 June 2007, pp. 1-6.

[7] N. K. Sharma, A. Ghosh and R. K. Varma, "A Novel Placement Strategy for Facts Controllers," IEEE Transactions on Power Delivery, Vol. 18, No. 3, 2003, pp. 982 987. doi:10.1109/TPWRD.2003.813874

[8] C. Schaffner and G. Andersson, "Performance of a TCSC for Congestion Relief," Proceedings of the Conference on Power Technology, St. Petersburg, 23-27 June, 2005, pp. 1-3.

[9] H. Besharat and S. A. Taher, "Congestion Management by Determining Optimal Location of TCSC in Deregulated Power Systems," International Journal of Electrical Power \& Energy Systems, Vol. 30, No. 10, 2008, pp. 563-568. doi:10.1016/j.ijepes.2008.08.007

[10] S. N. Singh and A. K. David, "Congestion Management by Optimizing FACTS Device Location," Proceedings of the International Conference on Electric Utility Deregulation and Restructuring and Power Technologies, London, 4-7 April 2000, pp. 23-28.

[11] S. A. Taher and H. Besharat, "Transmission Congestion Management by Determining Optimal Location of FACTS Devices in Deregulated Power Systems," American Journal of Applied Sciences, Vol. 5, No. 3, 2008, pp. 242-247.

[12] Y. Shi, K. Mwanza and L. A. Tuan, "Valuation of FACTS for Managing Congestion in Combined Pool and Bilateral Markets," Proceedings of Power Engineering Society Conference and Exposition in Africa, Johannesburg, 16-20 July 2007, pp. 1-7. doi:10.1109/PESAFR.2007.4498031

[13] N. Acharya and N. Mithulananthan, "Locating Series FACTS Devices for Congestion Managementin Deregulated Electricity Markets," Electric Power Systems Research, Vol. 77, No. 3-4, 2006, pp. 352-360. doi:10.1016/j.epsr.2006.03.016 